\title{
Tuning the magnetic properties of NiPS 3 through organic-ion intercalation
}

Daniel Tezze ${ }^{\mathrm{a}}$, José M. Pereira ${ }^{\mathrm{a}}$, Yaiza Asensio ${ }^{\mathrm{a}}$, Mihail Ipatov ${ }^{\mathrm{b}}$, Francesco Calavalle ${ }^{\mathrm{a}}$, Felix Casanova ${ }^{\mathrm{a}, \mathrm{c}}$, Alexander M. Bittner ${ }^{\mathrm{a}, \mathrm{c}}$, Maider Ormaza*d, Beatriz Martín-García*a, Luis E. Hueso ${ }^{\mathrm{a}, \mathrm{c}}$, Marco Gobbi*a,ce $^{*}$

a. CIC nanoGUNE BRTA, 20018 San Sebastian, Spain.

b. SGIker Medidas Magnéticas Gipuzkoa, UPV/EHU, 20018 San Sebastian, Spain.

c. IKERBASQUE, Basque Foundation for Science, 48013 Bilbao, Spain.

d. Departamento de Polímeros y Materiales Avanzados, Facultad de Químicas UPV/EHU

Apartado 1072, 20080 Donostia-San Sebastián, Spain.

e. $\quad$ Materials Physics Center CSIC-UPV/EHU, 20018 Donostia-San Sebastian, Spain.

Corresponding authors:

Maider Ormaza: maider.ormaza@ehu.es

Beatriz Martín-García: b.martingarcia@nanogune.eu

Marco Gobbi: m.gobbi@nanogune.eu

Atomically thin van der Waals magnetic crystals are characterized by tunable magnetic properties related to their low dimensionality. While electrostatic gating has been used to tailor their magnetic response, chemical approaches like intercalation remain largely unexplored. Here, we demonstrate the manipulation of the magnetism in the van der Waals antiferromagnet $\mathrm{NiPS}_{3}$ through the intercalation of different organic cations, inserted using an engineered two-step process. First, the electrochemical intercalation of tetrabutylammonium cations $\left(\mathrm{TBA}^{+}\right)$results in a ferrimagnetic hybrid compound displaying a transition temperature of $78 \mathrm{~K}$, and characterized by a hysteretic behavior with finite remanence and coercivity. Then, $\mathrm{TBA}^{+}$cations are replaced by cobaltocenium via an ion-exchange process, yielding a ferrimagnetic phase with higher transition temperature (98 K) and higher remanent magnetization. Importantly, we demonstrate that the intercalation and cation exchange processes can be carried out in bulk crystals and few-layer flakes, opening the way to the integration of intercalated magnetic materials in devices.

\section{Introduction}

The discovery of ferromagnetic order at the single-layer limit in $\mathrm{CrI}_{3}$ and $\mathrm{CrGeTe}_{3}{ }^{1,2}$ has motivated a sudden interest towards layered magnetic materials (LMMs), ${ }^{3,4}$ in which a van der Waals (vdW) gap separates sheets of covalently bound atoms. One of the distinctive features that differentiate ultrathin LMMs from 
conventional bulk magnetic compounds is the tunability of their magnetic properties, which stems from their reduced dimensionality and electronic properties. While several recent studies show how the magnetic response of LMMs can be significantly altered through electrostatic gating, ${ }^{5-9}$ the impact of chemical engineering approaches ${ }^{10-13}$ remains much less explored.

The process of inserting guest species into the vdW gaps of a host layered material ${ }^{14-17}$, known as intercalation, is a powerful route to manipulate the physical properties of layered materials. The occupation of the vdW gap typically leads to an expansion of the interlayer distance and a large charge transfer. ${ }^{18-26}$ Intercalation has been studied for several decades ${ }^{27,28}$ to induce superconductivity in layered materials ${ }^{18,20-}$ ${ }^{30}$, and it is subject of a recent renewed interest due to the possibility of tuning the properties of atomically thin micrometric flakes ${ }^{31,32}$ and tailoring the magnetism of $\mathrm{LMMs}^{29,33}$.

Among LMMs, NiPS 3 is a particularly intriguing compound, since it can be exfoliated to the single-layer limit and it exhibits an intralayer zig-zag antiferromagnetic order ${ }^{34}$ with a bulk Néel temperature of $155 \mathrm{~K}^{35}$. The transition temperature does not dramatically vary from bulk crystals to the few-layers limit, and the magnetic order remains down to the bilayer ${ }^{36}$. The effect of intercalation on the magnetism of $\mathrm{NiPS}_{3}$ has been so far studied by inserting different inorganic ${ }^{37,38}$ and organic ions ${ }^{39,40}$. In particular, wet chemical approaches were employed to intercalate $\mathrm{Li}^{+37,38}$, cobaltocene ${ }^{39}$, and 1,10-phenanthroline ${ }^{40}$ in $\mathrm{NiPS}_{3}$, resulting in paramagnetic ${ }^{41}$ or ferrimagnetic ${ }^{39,40}$ hybrid compounds. While these works demonstrate the potential of intercalation to alter the magnetism of layered materials, they do not fully exploit the chemical versatility of organic guest species and intercalating strategies to finely tune the magnetic properties in hybrid compounds.

Here, we demonstrate a controllable manipulation of the magnetic properties of $\mathrm{NiPS}_{3}$ through the intercalation of two different organic cations, inserted using an engineered two-step procedure. A first electrochemical process leads to the intercalation of tetrabutylammonium ions $\left(\mathrm{TBA}^{+}\right)$, which causes the emergence of ferrimagnetic ordering, characterized by a finite hysteresis and a transition temperature of 78 $\mathrm{K}$. Then, a novel cation exchange strategy yields the insertion of cobaltocenium ions $\left(\mathrm{Co}(\mathrm{Cp})_{2}{ }^{+}\right.$, where $\mathrm{Cp}$ is a cyclopentadienyl ring $\mathrm{C}_{5} \mathrm{H}_{5}{ }^{-}$), leading to a remarkable shift of the transition Curie temperature to $98 \mathrm{~K}$ and a higher remanent magnetization. Importantly, these processes were successfully carried out both in bulk crystals, as demonstrated by X-ray diffraction, and in mechanically exfoliated micrometric flakes, as monitored through micro-Raman spectroscopy. Our results open a novel route for the manipulation of the 2D magnetism of LMMs and their integration in devices.

For the insertion of $\mathrm{TBA}^{+}$cations in $\mathrm{NiPS}_{3}$, we employ an electrochemical approach, which typically provides a fast, controllable and reproducible intercalation ${ }^{16,42,43}$. We note that an electrochemical process was previously used to intercalate $\mathrm{NiPS}_{3}$ with alkali metals for Lithium-based batteries applications ${ }^{44}$ and to exfoliate it down to the monolayer using organic ions $s^{45-46}$, but not to produce bulk intercalated crystals. 
Fig. 1a presents a sketch of the setup used for the electrochemical process and the intercalating mechanism of $\mathrm{TBA}^{+}$in bulk NiPS 3 crystals (see also Fig. S1a in ESI section 1). At the cathodic side of the cell, a NiPS crystal with a typical mass in the range of $2-3 \mathrm{mg}$ is attached on a platinum plate using pressed indium dots. A silver plate serves as the anode. Both electrodes are immersed in a tetrabutylammonium bromide (TBAB) solution $(2 \mathrm{mg} / \mathrm{mL})$ under the strict absence of oxygen and water, and are connected to an external source-measure unit, which delivers a constant current through the electrochemical cell (see ESI Section $1)$.

When the current is applied, the $\mathrm{Br}^{-}$anions in solution move to the positively biased $\mathrm{Ag}$ anode, and the organic $\mathrm{TBA}^{+}$cations to the negatively biased $\mathrm{NiPS}_{3}$ crystal (Fig. 1a). At the anode, silver is oxidized, forming initially $\operatorname{AgBr}_{(\mathrm{s})}{ }^{20}$ :

$$
\begin{array}{lr}
\text { Oxidation of } \mathrm{Ag} & \mathrm{Ag} \rightarrow \mathrm{Ag}^{+}+\mathrm{e}^{-} \\
\text {Precipitation of } \mathrm{AgBr}_{(s)} & \mathrm{Ag}^{+}+\mathrm{Br}^{-} \rightarrow \mathrm{AgBr}
\end{array}
$$

Then $\operatorname{AgBr}_{(\mathrm{s})}$ undergoes subsequent complexation steps ${ }^{47}$.

At the cathode, electrons are injected into low-lying partially filled 3d-block electronic bands of $\mathrm{NiPS}_{3}{ }^{43}$. TBA ${ }^{+}$guest cationic species are electrostatically attracted into the vdW gaps to balance the total electric charge of the system:

$$
\begin{array}{ll}
e^{-} \text {injection into } \mathrm{NiPS}_{3} & \multicolumn{1}{c}{\mathrm{NiPS}_{3}+e^{-}} \\
& \\
& \mathrm{TBA}^{+} \\
\text {intercalation } & \left.\rightarrow \mathrm{NBP}_{3}\right)^{-}
\end{array}
$$

We found that the electrochemical process leads to an intercalated crystal that corresponds to a $\mathrm{TBA}_{0.25} \mathrm{NiPS}_{3}$ unit formula, where the 0.25 stoichiometric index is calculated through gravimetric analysis (see ESI Section 2).

Figure 1b displays a comparison between the XRD patterns measured for a pristine $\mathrm{NiPS}_{3}$ (see also Fig. S3, Table S1 in ESI section 3)) and an intercalated $\mathrm{TBA}_{0.25} \mathrm{NiPS}_{3}$ (also see Fig. S4a in ESI section 3). The untreated $\mathrm{NiPS}_{3}$ crystal exhibits sharp (001) peaks corresponding to a $6.34 \AA$ Anterlayer distance, in good agreement with the value reported in the literature ${ }^{48}$. After intercalation, the XRD pattern shows dramatic changes. First, the diffraction peaks (001) corresponding to the untreated crystal cannot be detected in 
$\mathrm{TBA}_{0.25} \mathrm{NiPS}_{3}$, evidencing the absence of regions characterized by the pristine interlayer spacing in the intercalated crystal, and confirming the complete intercalation. Moreover, new (001) diffraction peaks shifted to lower $2 \theta$ values emerge in the XRD pattern of $\mathrm{TBA}_{0.25} \mathrm{NiPS}_{3}$, corresponding to an increase of the interlayer distance due to the insertion of $\mathrm{TBA}^{+}$ions (Fig. 1c). At high $2 \theta$ angles, single peaks can be resolved in multiplets, revealing the presence of more than one crystallographic phase (see Table S2 in ESI section 3). We explain this feature by considering that the $\mathrm{TBA}^{+}$guest molecules can adopt different orientations with respect to the ab plane of $\mathrm{NiPS}_{3}$, leading to crystallographic phases characterized by a slightly different interlayer spacing. For the crystal shown in Fig. 1b, the most abundant phase is characterized by an interlayer distance of $15.0 \AA$. The other crystallographic phases encountered after TBA ${ }^{+}$ intercalation are characterized by slightly different interlayer distance (in the range $14.5 \AA-15.3 \AA$ ). The difference between the interlayer spacing measured for the intercalated and pristine device, which amounts to $8.2-8.9 \AA$ A depending on the crystallographic phase, provides an estimation of the vdW steric hindrance of the cations inserted in the host material. We note that by changing the conditions of the electrochemical intercalation, it is possible tune the relative abundance of the different crystallographic phases, as detailed in ig. S3 and Table S1 in ESI.

Based on the measured interlayer distance, the estimated size of the $\mathrm{TBA}^{+}$cations ${ }^{49}$ and the density of $\mathrm{TBA}^{+}$ molecules in the organic layer (one molecule every four formula units), we can conclude that the intercalation leads to an organic-inorganic hybrid superlattice composed of alternated single $\mathrm{TBA}^{+}$organic layers and $\mathrm{NiPS}_{3}$ monolayers (Fig. 1c).

By comparing the XRD pattern of $\mathrm{TBA}_{0.25} \mathrm{NiPS}_{3}$ with those of other electrochemically intercalated compounds ${ }^{50,51}$, we find that the $\mathrm{TBA}_{0.25} \mathrm{NiPS}_{3}$ peaks are relatively sharp and defined, indicating a relative high crystalline quality.

Next, we show that the substitution of $\mathrm{TBA}^{+}$guest ions with $\mathrm{Co}(\mathrm{Cp})_{2}{ }^{+}$ions through a non-redox heterogeneous cation exchange mechanism in solution (Fig. 2a). The mechanism can be described as follows:

$$
\begin{aligned}
4 T B A_{0.25} \mathrm{NiPS}_{3}+\mathrm{Co}(\mathrm{Cp})_{2}{ }^{+} & \\
& \rightarrow 4\left[\mathrm{Co}(\mathrm{Cp})_{2}\right]_{0.25} \mathrm{NiPS}_{3}+\mathrm{TBA}^{+}
\end{aligned}
$$

To the best of our knowledge, this method has not been employed until now to tune the physical properties of an already intercalated $\mathrm{NiPS}_{3}$ crystal. Pioneering studies on the $\mathrm{NiPS}_{3}$ intercalation show how a solvothermal method results in the direct insertion $\mathrm{Co}(\mathrm{Cp})_{2}{ }^{+}$cations; however, this study only reported successful intercalation of powder at $130{ }^{\circ} \mathrm{C} .{ }^{39}$ Our protocol follows a 2-step intercalating route that allows 
large $\left(0.1 \mathrm{~mm} 5 \mathrm{~mm}^{2}\right) \mathrm{NiPS}_{3}$ crystals (typically $2-3 \mathrm{mg}$ ) to be intercalated with $\mathrm{Co}(\mathrm{Cp})_{2}{ }^{+}$cations in a similarly long process, even at room temperature.

In Fig. 2b, the diffractogram of $\left[\mathrm{Co}(\mathrm{Cp})_{2}\right]_{0.25} \mathrm{NiPS}_{3}$ (see also Fig. S5a and Table S3 in ESI section 3) is compared with the previous one of $\mathrm{TBA}_{0.25} \mathrm{NiPS}_{3}$. After the ion exchange, the peaks corresponding to the $\mathrm{TBA}_{0.25} \mathrm{NiPS}_{3}$ phase are absent, indicating that the $\mathrm{TBA}^{+}$cations have been successfully de-intercalated. Additionally, the (001) diffraction peaks located at higher angles evidence a shorter interlayer distance (Fig. 2b). This observation is in agreement with the smaller size of $\mathrm{Co}(\mathrm{Cp})_{2}{ }^{+52}$ as compared to $\mathrm{TBA}^{+}$. This comparison confirms the occurrence of a complete $\mathrm{TBA}^{+} / \mathrm{Co}(\mathrm{Cp})_{2}{ }^{+}$exchange.

Interestingly, the XRD pattern of $\left[\mathrm{Co}(\mathrm{Cp})_{2}\right]_{0.25} \mathrm{NiPS}_{3}$ includes two families of peaks associated with two slightly different phases with different interlayer distances, $11.9 \AA$, and $11.5 \AA$. These two different gaps correspond to an increase in the separation between layers compared to the pristine material of $5.6 \AA$ and $5.2 \AA$, respectively. We understand this phenomenon as the consequence of the coexistence of two possible arrangements for the $\mathrm{Co}(\mathrm{Cp})_{2}{ }^{+}$ions within the vdW gap, i.e., horizontally and vertically, with the the $\mathrm{C}_{5}$ symmetry axis of $\mathrm{Cp}^{-}$respectively parallel and perpendicular to the basal plane of the crystal. A $5.2 \AA$ increase in interlayer distance was already reported for the intercalation of $\mathrm{Co}(\mathrm{Cp})_{2}{ }^{+}$in $\mathrm{MnPS}_{3}$ via cationic exchange $^{53}$, for which it was revealed that the $\mathrm{Co}(\mathrm{Cp})_{2}{ }^{+}$is horizontally oriented (as shown in Fig. 2c). Therefore, we assign the $5.6 \AA$ and $5.2 \AA$ phases encountered in our sample to $\mathrm{Co}(\mathrm{Cp})_{2}{ }^{+}$cations oriented

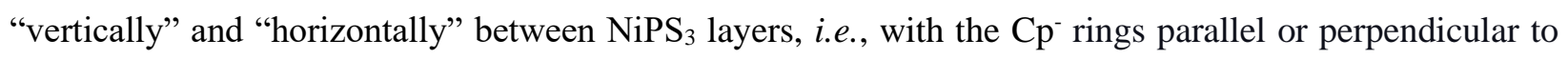
the basal plane, respectively. Notice here that a more complex assembly of vertical and horizontal cations cannot be excluded in the $5.6 \AA$ phase, as reported for neutral metallocenes on metallic surfaces ${ }^{54}$. Broadening of the peaks remains almost unaltered (see Table S3 in ESI section 3), indicating that the cation exchange process, even if highly invasive, does not affect the macroscopic lattice crystallinity. We note that by employing different conditions for the intercalation, we obtained a $\mathrm{Co}(\mathrm{Cp})_{2}{ }^{+}$intercalated $\mathrm{NiPS}_{3}$ displaying the single structural phase characterized by the $11.9 \AA$ Anterlayer distance (see Fig. S5 and Table $\mathrm{S} 3$ in ESI).

After demonstrating the $\mathrm{TBA}^{+}$intercalation and the $\mathrm{TBA}^{+} / \mathrm{Co}(\mathrm{Cp})_{2}{ }^{+}$exchange through $\mathrm{XRD}$, we now discuss how micro-Raman spectroscopy can be exploited as a sensitive tool to monitor the intercalating process and to provide additional information on the phenomena accompanying the insertion of cations. The Raman spectrum of pristine $\mathrm{NiPS}_{3}$ (black curve in Fig. 3a) displays several peaks, which correspond to the eight fundamental $\left(3 \mathrm{~A}_{1 \mathrm{~g}}+5 \mathrm{E}_{\mathrm{g}}\right)$ and some higher order Raman-active modes. ${ }^{55}$

The Raman spectrum of $\mathrm{TBA}_{0.25} \mathrm{NiPS}_{3}$ (red curve in the middle panel of Fig. 3a) is significantly different from that of the pristine $\mathrm{NiPS}_{3}$. We highlight three main changes occurring after the $\mathrm{TBA}^{+}$insertion which can be considered the hallmarks of successful intercalation. More detailed Raman spectra are reported in Fig. S7 and other features are present in ESI section 3. First, the intensity of the $\mathrm{E}_{\mathrm{g}}{ }^{(1)}$ peak at $132 \mathrm{~cm}^{-1}$, 
which is related to the translational mode of $\mathrm{Ni}$ (II) cations in sulfur-coordinated octahedral sites $\left(O_{h}\right)^{48}$, is strongly suppressed for $\mathrm{TBA}_{0.25} \mathrm{NiPS}_{3}$. A similar effect was previously reported for $\mathrm{Li}^{+}$intercalated $\mathrm{NiPS}_{3}{ }^{56}$, and attributed to a lower density of $\mathrm{Ni}(\mathrm{II})$ in $O_{h}$ sites. Indeed, $\mathrm{Ni}$ atoms are reduced to the metallic oxidation state $\left(\mathrm{Ni}^{0}\right)$ and displaced to the tetrahedral sites $\left(T_{d}\right)^{37,57}$. Therefore, the decrease in intensity of the $\mathrm{E}_{\mathrm{g}}{ }^{(1)}$ mode provides the first direct evidence of the $\mathrm{Ni}(\mathrm{II}) \rightarrow \mathrm{Ni}^{0}$ coordination evolution induced by the intercalation.

Second, three new peaks (indicated with ML in Fig. 3a) appear at $202 \mathrm{~cm}^{-1}, 618 \mathrm{~cm}^{-1}$ and $635 \mathrm{~cm}^{-1}$ in the Raman spectrum of $\mathrm{TBA}_{0.25} \mathrm{NiPS}_{3}$. Similar spectral features were observed for ultrathin $\mathrm{NiPS}_{3}$ flakes, and their presence was explained based on the breaking of translational symmetry ${ }^{36,55}$. The emergence of these peaks in $\mathrm{TBA}_{0.25} \mathrm{NiPS}_{3}$ indicates that the increased interlayer distance in the intercalated crystals produces a symmetry breaking effect similar to that generated by the isolation of monolayers. In this regard, the observation of vibrational features typical of monolayers being recovered in intercalated crystals shows the potential of intercalation to obtain monolayer-like behavior in bulk crystals. ${ }^{31}$

Lastly, we highlight the remarkable position change of the $\mathrm{A}_{\mathrm{lg}}{ }^{(1)}$ peak, which shifts from $254.5 \mathrm{~cm}^{-1}$ in the pristine $\mathrm{NiPS}_{3}$ to $245 \mathrm{~cm}^{-1}$ in $\mathrm{TBA}_{0.25} \mathrm{NiPS}_{3}$. A similar redshift but larger for $\mathrm{A}_{1 \mathrm{~g}}{ }^{(1)}$ was reported for $\mathrm{Li}^{+}$ intercalated $\mathrm{NiPS}_{3}$, attributed to the charge carrier doping introduced by intercalation. ${ }^{56}$ This can be understood as a result of the lower doping level introduced by the $\mathrm{TBA}^{+}$ions intercalation, that is limited by their higher molecular vdW hindrance.

These three main features also appear in the Raman spectrum of the $\left[\mathrm{Co}(\mathrm{Cp})_{2}\right]_{0.25} \mathrm{NiPS}_{3}$ (blue curve in the bottom panel of Fig. 3a). In addition to these changes, for this sample we also observe the peaks related to the $v\left(C o-C_{5}\right), \delta_{/ /}(C-H), v(C-C)$ molecular modes of $\mathrm{Co}(\mathrm{Cp})_{2}{ }^{+}$, located at $314 \mathrm{~cm}^{-1}, 1062 \mathrm{~cm}^{-1}$ and $1108 \mathrm{~cm}^{-1}$, respectively. These peaks are red-shifted to those observed for the $\mathrm{Co}(\mathrm{Cp})_{2} \mathrm{PF}_{6}$ powder, located at $317 \mathrm{~cm}^{-1}, 1070 \mathrm{~cm}^{-1}$ and $1112.5 \mathrm{~cm}^{-1}$ (see Fig. S7 in ESI) ${ }^{58}$ This indicates that $\mathrm{Co}(\mathrm{Cp})_{2}{ }^{+}$ions are structurally intact and surrounded by a different chemical environment than the one provided by the coordinating $\mathrm{PF}_{6}{ }^{-}$in the pristine crystal lattice. Moreover, we also highlight that the main Raman feature of $\mathrm{PF}_{6}{ }^{-}$counterion ${ }^{59}, v(P-F)$ at $741 \mathrm{~cm}^{-1}$, is not detected in $\left[\mathrm{Co}(\mathrm{Cp})_{2}\right]_{0.25} \mathrm{NiPS}_{3}$. This proves that the cation exchange effectively results in the swapping of $\mathrm{TBA}^{+}$with $\mathrm{Co}(\mathrm{Cp})_{2}{ }^{+}$, and that the $\mathrm{Co}(\mathrm{Cp})_{2}{ }^{+}$features detected in the crystals are not parasitic $\mathrm{Co}(\mathrm{Cp})_{2} \mathrm{PF}_{6}$ vibrational signatures appearing due to the presence of residuals of the salt.

Our analysis demonstrates that Raman spectroscopy is a suitable tool for characterization of the intercalation process in bulk crystals; therefore, we employ it to monitor the intercalation of micrometric mechanically exfoliated flakes. This is of particular significance, since other techniques employed to track the intercalation of bulk crystals (such as XRD) are not practicable for the characterization of few-layersthick and few-micrometres-wide exfoliated flakes. 
To intercalate micrometric $\mathrm{NiPS}_{3}$ crystals, we stamped a mechanically exfoliated flake onto prepatterned Au contacts (see ESI Section 1). After that, we performed the electrochemical intercalation of $\mathrm{TBA}^{+}$cations by contacting one of the electrodes to the external circuitry, as shown in Fig. 3b (real apparatus shown in Fig. S1c in ESI section 1). Subsequently, the $\mathrm{TBA}^{+} \rightarrow \mathrm{Co}(\mathrm{Cp})_{2}{ }^{+}$cation exchange was achieved by simply drop-casting a $\mathrm{Co}(\mathrm{Cp})_{2} \mathrm{PF}_{6}$ solution onto the $\mathrm{TBA}^{+}$intercalated flake under strict exclusion of oxygen and water. In Fig. 3b, we compare the Raman spectra measured (i) on a pristine flake transferred onto prepatterned contacts, (ii) on the same flake after the electrochemical intercalation of $\mathrm{TBA}^{+}$cations, and (iii) for the same flake after the $\mathrm{TBA}^{+} / \mathrm{Co}(\mathrm{Cp})_{2}{ }^{+}$cation exchange. The Raman spectrum of the pristine flake is analogous to that of the untreated bulk crystal, displaying all the Raman-active modes. The peaks of the exfoliated flake are generally broader than those of the bulk crystal, which can be ascribed to lattice strain due to the stamping ${ }^{60}$, or interaction with the $\mathrm{SiO}_{2}$ substrate ${ }^{61}$. Since the flake is thin, the typical Raman features of the silicon substrate appear at $303 \mathrm{~cm}^{-1}, 520 \mathrm{~cm}^{-1}$ and around $960 \mathrm{~cm}^{-1}$. Remarkably, after intercalating the flake with $\mathrm{TBA}^{+}$, we observe the same modifications found in the Raman spectrum previously described for bulk crystals - including the lower intensity in the $\mathrm{Ni}$ (II) vibrational mode $\mathrm{E}_{\mathrm{g}}{ }^{(1)}$, the emergence of new peaks characteristic of the reduced symmetry, and the doping-related redshift in $\mathrm{A}_{1 \mathrm{~g}}{ }^{(1)}$. Moreover, even after the cation exchange, we observe the spectral features discussed for its corresponding bulk crystal case, which include the appearance of the characteristic vibrational modes of $\mathrm{Co}(\mathrm{Cp})_{2}{ }^{+}$.

These results demonstrate that (i) the electrochemical and ion exchange processes developed here lead to efficient intercalation not only in bulk crystals, but also in mechanically exfoliated flakes, and that (ii) micro-Raman spectroscopy is an ideal technique to monitor the intercalation process for ultrathin micrometric exfoliated flakes.

Finally, we show how the intercalation and ion exchange processes modify the magnetic properties of $\mathrm{NiPS}_{3}$. Figure 4a shows the temperature dependence of the field-cooled magnetization $\mathrm{M}(\mathrm{T})$ measured within an in-plane magnetic field $\mathrm{H}=500 \mathrm{Oe}$ for pristine, $\mathrm{TBA}^{+}$and $\mathrm{Co}(\mathrm{Cp})_{2}{ }^{+}$intercalated $\mathrm{NiPS}_{3}$. For the pristine $\mathrm{NiPS}_{3}$ crystal, the $\mathrm{M}(\mathrm{T})$ displays the reported trend ${ }^{34,36}$, characterized by a decrease in the magnetization associated with the antiferromagnetic transition at $\mathrm{T}_{\text {Néel }}=155 \mathrm{~K}$. A very different behavior is observed for $\mathrm{TBA}^{+}$and $\mathrm{Co}(\mathrm{Cp})_{2}{ }^{+}$intercalated $\mathrm{NiPS}_{3}$. In both cases, we find a sudden increase of the magnetization at $78 \mathrm{~K}$ for $\mathrm{TBA}_{0.25} \mathrm{NiPS}_{3}$ and at $98 \mathrm{~K}$ for $\left[\mathrm{Co}(\mathrm{Cp})_{2}\right]_{0.25} \mathrm{NiPS}_{3}$, characteristic of a magnetic phase transition. The magnetization values achieved at low temperatures, which are for both compounds on the order of $10^{-3} \mu_{\mathrm{B}}$ per $\mathrm{Ni}$ atom, are low compared to the expected magnetic moment of $\mathrm{Ni}(\mathrm{II})(\mu[\mathrm{Ni}(\mathrm{II})]$ $\left.=2.83 \mu_{\mathrm{B}}\right)$. Therefore, we conclude that at low-temperature $\mathrm{TBA}_{0.25} \mathrm{NiPS}_{3}$ and $\left[\mathrm{Co}(\mathrm{Cp})_{2}\right]_{0.25} \mathrm{NiPS}_{3}$ are not ferromagnetic, but rather ferrimagnetic. Moreover, in the $\mathrm{M}(\mathrm{T})$ we observe an upturn of the magnetization below $20 \mathrm{~K}$ for both intercalated compounds, suggesting the co-existence of paramagnetic and 
ferrimagnetic phases. We note that the magnetization reached at low temperature for the two compounds is quite different, being $7.5 \mathrm{emu} / \mathrm{mol}(\mathrm{Ni})$ for $\mathrm{TBA}_{0.25} \mathrm{NiPS}_{3}$ and $20 \mathrm{emu} / \mathrm{mol}(\mathrm{Ni})$ for $\left[\mathrm{Co}(\mathrm{Cp})_{2}\right]_{0.25} \mathrm{NiPS}_{3}$, indicating that the latter is characterized by a larger spontaneous magnetization (see also Fig. S8 in ESI). This scenario is confirmed by the hysteresis loops $\mathrm{M}(\mathrm{H})$ measured at $5 \mathrm{~K}$ for $\mathrm{TBA}^{+}$and $\mathrm{Co}(\mathrm{Cp})^{+}$intercalated $\mathrm{NiPS}_{3}$ displayed in Fig. 4b, compared with the loop recorded for pristine NiPS 3 . Magnetization of pristine $\mathrm{NiPS}_{3}$ increases linearly with the applied field $\mathrm{H}$. This trend can be understood considering that $\mathrm{NiPS}_{3}$ is an antiferromagnet with an in-plane anisotropy, and that the application of an in-plane field causes a slight tilting of the spins along its direction. Conversely, the $\mathrm{M}(\mathrm{H})$ curves of $\mathrm{TBA}_{0.25} \mathrm{NiPS}_{3}$ and $\left[\mathrm{Co}(\mathrm{Cp})_{2}\right]_{0.25} \mathrm{NiPS}_{3}$ show a clear hysteresis with finite coercive fields of $\mathrm{H}=1.5 \mathrm{kOe}$ and $2.2 \mathrm{kOe}$, as well as remanent magnetization $\mathrm{M}_{\mathrm{r}}=6.6 \mathrm{emu} / \mathrm{mol}$ and $14.2 \mathrm{emu} / \mathrm{mol}$, respectively. In both cases, the hysteretic behavior demonstrates the emergence of (ferri)magnetic ordering. At large magnetic fields, the magnetization does not saturate, confirming the presence of a paramagnetic signal in addition to the ferrimagnetic one. The coexistence of different magnetic responses can be ascribed to the different structural phases found in our intercalated samples. This conclusion is supported by a combined structural and magnetic characterization of other crystals intercalated using different conditions (see ESI Section 7). In particular, the magnetic response in $\mathrm{TBA}^{+}$intercalated $\mathrm{NiPS}_{3}$ was found to depend on the details of the structural phases generated in the intercalation process. Besides, the single-phase $\left[\mathrm{Co}(\mathrm{Cp})_{2}\right]_{0.25} \mathrm{NiPS}_{3}$ crystal characterized by the 11.9 $\AA$ interlayer distance did not display the ferrimagnetic behavior (Fig. S9 in ESI). Therefore, we conclude that for this compound, the paramagnetic and the ferromagnetic phases are related to the phases with 11.9 $\AA$ and $11.5 \AA$ Anterlayer distance, respectively.

We note that (i) the ferrimagnetism in $\mathrm{TBA}^{+}$intercalated $\mathrm{NiPS}_{3}$, which has not been previously reported, proves that an electrochemical approach can be used to modulate the magnetism of $\mathrm{NiPS}_{3}$; and (ii) the phase transition at $98 \mathrm{~K}$ measured in our $\mathrm{Co}(\mathrm{Cp})_{2}$-intercalated $\mathrm{NiPS}_{3}$ is significantly higher than the one previously reported for the same compound intercalated using a wet chemistry approach ${ }^{39}$. This indicates that not only the guest molecule, but also the intercalation method can strongly impact the resulting magnetic properties of $\mathrm{NiPS}_{3}$.

The dramatic change in the magnetic properties of $\mathrm{NiPS}_{3}$ can be explained based on the nanoscale phenomena taking place in the $\mathrm{NiPS}_{3}$ layers. In particular, the intercalation induces the $\mathrm{Ni}(\mathrm{II}) \rightarrow \mathrm{Ni}^{0}$ reduction and displacement from the $T_{d}$ to the $O_{h}$ lattice site ${ }^{37,57}$ (see Fig. $4 \mathbf{c}$ ). This reductive displacement is accompanied by a dramatic change in the magnetic properties of the $\mathrm{Ni}$ atoms. $\mathrm{Ni}(\mathrm{II})-O_{h}$ atoms are characterized by a $[\mathrm{Ar}] 3 \mathrm{~d}^{8} \mathrm{~s}^{0}$ electronic configuration bearing a magnetic moment $\mu[\mathrm{Ni}(\mathrm{II})]=2.83 \mu_{\mathrm{B}}$, whereas $\mathrm{Ni}^{0}-T_{d}$ possess a $[\mathrm{Ar}] 3 \mathrm{~d}^{10} \mathrm{~s}^{0}$ zero-spin (diamagnetic) configuration. As discussed previously, Raman spectroscopy evidences that the $\mathrm{Ni}(\mathrm{II})-O_{h} \rightarrow \mathrm{Ni}^{0}-T_{d}$ reduction occurs for a significant fraction of $\mathrm{Ni}(\mathrm{II})$ atoms, which can be quantified based on charge balance considerations. The reduction of each $\mathrm{Ni}$ (II) atom 
requires two transferred electrons, which are provided by two guest molecules. Therefore, from the 0.25 stoichiometric index in $\mathrm{TBA}_{0.25} \mathrm{NiPS}_{3}$, we estimate that $12.5 \%$ of $\mathrm{Ni}$ atoms are reduced and displaced. As a result, the antiferromagnetism in the intercalated compounds is not fully compensated, as part of the spins that give rise to the antiferromagnetic order in the pristine crystal are suppressed during the intercalation, or analogously, unpaired spins are introduced in the system (see Fig. 4c).

While this argument accounts for the presence of unpaired spins in the intercalated $\mathrm{NiPS}_{3}$ compounds, understanding their magnetic coupling is more complex. Our $\mathrm{M}(\mathrm{T})$ and $\mathrm{M}(\mathrm{H})$ data indicate that different structural phases of crystals intercalated with the same molecule are characterized by diverse magnetic responses, demonstrating that the overall magnetic properties are determined by the details of the nanoscale interactions between the guest species and the inorganic layers. Therefore, our results indicate that the intercalation of organic cations provides a readily available "tuning wheel" to engineer the magnetic properties of layered materials. 


\section{Materials and methods}

\section{Materials}

$\mathrm{NiPS}_{3}$ crystals were purchased from HQ graphene. Acetonitrile (anhydrous $<0.001 \% \mathrm{H}_{2} \mathrm{O}$ ), tetrabuthyl-ammonium bromide (TBAB - purity $\geq 98 \%$ ) and bis-(cyclopentadienyl)cobalt(III) hexafluorophosphate $\left(\mathrm{Co}(\mathrm{Cp})_{2} \mathrm{PF}_{6}\right.$ - purity $\left.98 \%\right)$ were purchased at Aldrich. Platinum and silver electrode plates were obtained by pressing metallic pellets (purity $99.99 \%$, Kurt J. Lesker). Before usage, salts were dehydrated at $100^{\circ} \mathrm{C}$ in vacuum $(1 \mathrm{mbar})$ overnight. Electrodes were polished with sandpaper and sonicated for 2 minutes in acetone and then in isopropanol for surface cleaning.

\section{Device fabrication}

To intercalate $\mathrm{NiPS}_{3}$ flakes, devices have been fabricated by stamping mechanically exfoliated flakes onto gold contacts prepatterned in a Hall configuration on $\mathrm{Si} / \mathrm{SiO}_{2}$ substrates. The stamping is performed by exfoliating $\mathrm{NiPS}_{3}$ on a polydimethylsiloxane (PDMS) elastomer. Target flakes with a thickness of a few layers (typically 5-15) are identified through optical microscopy and transferred onto the prepatterned contacts using a micromanipulator coupled to an optical microscope.

\section{$\mathrm{TBA}^{+}$electrochemical intercalation}

Self-limiting tetrabutylammonium $\left(\mathrm{TBA}^{+}\right)$electrochemical intercalation is achieved using a custom-made cell. This cell is composed of two electrodes, a platinum holder for $\mathrm{NiPS}_{3}$ as the cathode and a silver plate as the anode, both immersed in a TBAB solution in acetonitrile $(2 \mathrm{mg} / \mathrm{mL}-3.1 \mathrm{mM})$ (Fig. 1a).

Bulk NiPS 3 crystals (typical mass $\mathrm{m}=2-3 \mathrm{mg}$ ) were electrically anchored to the platinum plate by utilizing indium strips and subsequently fully dipped into the electrolyte. In this case, the intercalation was achieved by applying a constant current $(30 \mu \mathrm{A}$ or $50 \mu \mathrm{A})$ using a Keithley 2635 source meter. The voltage drop caused by the current was monitored during the intercalation, and the full intercalation was identified as an increase in the voltage (see also ESI 1). Micrometric flakes were intercalated by connecting one of the $\mathrm{Au}$ electrodes to the external circuitry using a $\mathrm{Cu}$ wire attached through an indium connection. In this case, the intercalation was achieved by applying a constant current of $2 \mu \mathrm{A}$ for 5 minutes.

\section{$\mathrm{TBA}^{+} / \mathrm{Co}(\mathrm{Cp})_{2}{ }^{+}$cation exchange}

Bulk TBA ${ }_{0.25} \mathrm{NiPS}_{3}$ crystal was immersed in $1 \mathrm{~mL}$ of $\mathrm{Co}(\mathrm{Cp})_{2} \mathrm{PF}_{6}$ solution in acetonitrile at a concentration of $50 \mathrm{mg} / \mathrm{mL}$. The process was carried out in a sealed vial kept in an oven at $50{ }^{\circ} \mathrm{C}$ located in a glovebox for 2 days to ensure the exchange for the whole crystal (see also ESI 1).

\section{Characterizations}

$\mathrm{X}$-ray diffractometry is carried out with an XPERT-PRO diffractometer on bulk crystals in a $\omega / \omega$ configuration. A copper cathode $\lambda\left(\mathrm{K}_{\alpha 1}\right)=1.540598 \AA$ is used as X-ray source. Micro-Raman characterization is performed with a Renishaw inVia Qontor system at room temperature using a $633 \mathrm{~nm}$ $\mathrm{HeNe}$ laser. Magnetization measurements vs. temperature or field $\mathrm{H}$ are carried out using a physical properties measurement system (PPMS) in vibrating sample magnetometer mode. 


\section{Conclusions}

We have employed an electrochemical approach to intercalate $\mathrm{TBA}^{+}$cations in the $\mathrm{LMM} \mathrm{NiPS}_{3}$, leading to a hybrid organic/inorganic superlattice compound composed of alternating $\mathrm{TBA}^{+}$and $\mathrm{NiPS}_{3}$ single layers. Moreover, immersing the $\mathrm{TBA}^{+}$intercalated crystals in a $\mathrm{Co}(\mathrm{Cp})_{2} \mathrm{PF}_{6}$ solution results in the complete exchange of the $\mathrm{TBA}^{+}$cations with $\mathrm{Co}(\mathrm{Cp})_{2}{ }^{+}$.

These processes, which are confirmed through XRD for large crystals, can be also monitored through Raman spectroscopy, which provides important insight into the nanoscale phenomena accompanying the intercalation. Importantly, we used micro-Raman spectroscopy to demonstrate the successful intercalation of micrometric exfoliated flakes, which could not be accessed via conventional XRD. Finally, we show that the magnetic properties of $\mathrm{NiPS}_{3}$ are dramatically modified by the intercalation in a way that depends on the molecular guests and the intercalation process. In particular, pristine $\mathrm{NiPS}_{3}$ is antiferromagnetic, whereas a ferrimagnetic transition at $78 \mathrm{~K}$ and $98 \mathrm{~K}$ is recorded in $\mathrm{TBA}^{+}$and $\mathrm{Co}(\mathrm{Cp})_{2}{ }^{+}$intercalated compounds, respectively. Our results show how molecular intercalation can be tailored to generate hybrid superlattice compounds with novel magnetic properties, offering the possibility to introduce additional compounds with tunable magnetic properties in the LMM family. Moreover, the successful intercalation of exfoliated flakes opens the way to the integration of intercalated $\mathrm{NiPS}_{3}$ in devices.

\section{Conflicts of interest}

There are no conflicts to declare.

\section{Author Contribution}

D. T., M.O, M.G. conceived the study. D.T. and A. B. performed the electrochemical intercalation, the ionexchange and XRD measurements. J.M.P., Y.A. and F. Calavalle fabricated the devices for the intercalation of flakes. J.M.P. and B. M.-G. performed the Raman characterization. M.I. carried out the magnetic measurements. M.G., B. M.-G. and L.E.H. supervised the work. D.T. and M.G. wrote the manuscript, with inputs from all co-authors.

\section{Acknowledgements}

The authors acknowledge R. Llopis and A. Eleta for technical support. This work is supported by "la Caixa" Foundation (ID 100010434), under the agreement LCF/BQ/PI19/11690017, by the Spanish MICINN under Project PID2019-108153GA-I00, RTI2018-094861-B-100 and under the María de Maeztu Units of Excellence Program (MDM-2016-0618). B.M-G. thanks Gipuzkoa Council (Spain) in the frame of Gipuzkoa Fellows Program.

\section{References}

1 B. Huang, G. Clark, E. Navarro-Moratalla, D. R. Klein, R. Cheng, K. L. Seyler, D. Zhong, E. Schmidgall, M. A. McGuire, D. H. Cobden, W. Yao, D. Xiao, P. Jarillo-Herrero and X. Xu, Nature, 2017, 546, 270-273.

2 C. Gong, L. Li, Z. Li, H. Ji, A. Stern, Y. Xia, T. Cao, W. Bao, C. Wang, Y. Wang, Z. Q. Qiu, R. J. Cava, S. G. Louie, J. Xia and X. Zhang, Nature, 2017, 546, 265-269.

3 M. Gibertini, M. Koperski, A. F. Morpurgo and K. S. Novoselov, Nature Nanotechnology, 2019, 14, 408-419.

4 M. Och, M.-B. Martin, B. Dlubak, P. Seneor and C. Mattevi, Nanoscale, 2021, 13, 2157-2180.

5 Y. Deng, Y. Yu, Y. Song, J. Zhang, N. Z. Wang, Z. Sun, Y. Yi, Y. Z. Wu, S. Wu, J. Zhu, J. Wang, X. H. Chen and Y. Zhang, Nature, 2018, 563, 94-99.

6 S. Jiang, J. Shan and K. F. Mak, Nat Mater, 2018, 17, 406-410.

7 S. Jiang, L. Li, Z. Wang, K. F. Mak and J. Shan, Nat Nanotechnol, 2018, 13, 549-553.

8 Z. Wang, T. Zhang, M. Ding, B. Dong, Y. Li, M. Chen, X. Li, J. Huang, H. Wang, X. Zhao, Y. Li, D. Li, C. Jia, L. Sun, H. Guo, Y. Ye, D. Sun, Y. Chen, T. Yang, J. Zhang, S. Ono, Z. Han and Z. Zhang, Nature Nanotechnology, 2018, 13, 554-559.

9 I. A. Verzhbitskiy, H. Kurebayashi, H. Cheng, J. Zhou, S. Khan, Y. P. Feng and G. Eda, Nature Electronics, 2020, 3, 460-465. 
10 D. Voiry, A. Goswami, R. Kappera, C. de C. C. e Silva, D. Kaplan, T. Fujita, M. Chen, T. Asefa and M. Chhowalla, Nature Chemistry, 2015, 7, 45-49.

11 L. Daukiya, J. Teyssandier, S. Eyley, S. El Kazzi, M. C. Rodríguez González, B. Pradhan, W.

Thielemans, J. Hofkens and S. De Feyter, Nanoscale, 2021, 13, 2972-2981.

12 I. Gómez-Muñoz, S. Laghouati, R. Torres-Cavanillas, M. Morant-Giner, N. V. Vassilyeva, A.

Forment-Aliaga and M. Giménez-Marqués, ACS Appl. Mater. Interfaces, 2021, 13, 36475-36481.

13 M. Gobbi, E. Orgiu and P. Samori, Advanced Materials, 2018, 30.

14 J. Wan, S. D. Lacey, J. Dai, W. Bao, M. S. Fuhrer and L. Hu, Chemical Society Reviews, 2016, 45, 6742-6765.

15 M. S. Stark, K. L. Kuntz, S. J. Martens and S. C. Warren, Advanced Materials, 2019, 31, 1808213.

16 J. Zhou, Z. Lin, H. Ren, X. Duan, I. Shakir, Y. Huang and X. Duan, Advanced Materials, 2021, 33, 2004557.

17 S. J. Sung, J. W. Yang, P. R. Lee, J. G. Kim, M. T. Ryu, H. M. Park, G. Lee, C. C. Hwang, Kwang. S. Kim, J. S. Kim and J. W. Chung, Nanoscale, 2014, 6, 3824-3829.

18 C. A. Formstone, E. T. FitzGerald, D. O’Hare, P. A. Cox, M. Kurmoo, J. W. Hodby, D. Lillicrap and M. Goss-Custard, J. Chem. Soc., Chem. Commun., 1990, 501-503.

19 Z. Li, Y. Zhao, K. Mu, H. Shan, Y. Guo, J. Wu, Y. Su, Q. Wu, Z. Sun, A. Zhao, X. Cui, C. Wu and Y. Xie, J. Am. Chem. Soc., 2017, 139, 16398-16404.

20 L. K. Ma, M. Z. Shi, B. L. Kang, K. L. Peng, F. B. Meng, C. S. Zhu, J. H. Cui, Z. L. Sun, D. H. Ma, H. H. Wang, B. Lei, T. Wu and X. H. Chen, Physical Review Materials, 2020, 4, 124803.

21 H. Wu, S. Li, M. Susner, S. Kwon, M. Kim, T. Haugan and B. Lv, 2D Materials, 2019, 6, 045048.

22 M. Z. Shi, N. Z. Wang, B. Lei, C. Shang, F. B. Meng, L. K. Ma, F. X. Zhang, D. Z. Kuang and X. H. Chen, Physical Review Materials, 2018, 2, 074801.

23 B. Rendenbach, T. Hohl, S. Harm, C. Hoch and D. Johrendt, J. Am. Chem. Soc., 2021, 143, 30433048.

24 M. Z. Shi, N. Z. Wang, B. Lei, J. J. Ying, C. S. Zhu, Z. L. Sun, J. H. Cui, F. B. Meng, C. Shang, L. K. Ma and X. H. Chen, New Journal of Physics, 2018, 20, 123007.

25 N. Z. Wang, M. Z. Shi, C. Shang, F. B. Meng, L. K. Ma, X. G. Luo and X. H. Chen, New Journal of Physics, 2018, 20, 023014.

26Z. Li, X. Zhang, X. Zhao, J. Li, T. S. Herng, H. Xu, F. Lin, P. Lyu, X. Peng, W. Yu, X. Hai, C. Chen, H. Yang, J. Martin, J. Lu, X. Luo, A. H. Castro Neto, S. J. Pennycook, J. Ding, Y. Feng and J. Lu, Advanced Materials, 2020, 32, 1907645.

27 Y. Koike, H. Suematsu, K. Higuchi and S. Tanuma, Solid State Communications, 1978, 27, 623-627.

28 T. E. Weller, M. Ellerby, S. S. Saxena, R. P. Smith and N. T. Skipper, Nature Physics, 2005, 1, $39-41$.

29 Z. Li, X. Zhang, X. Zhao, J. Li, T. S. Herng, H. Xu, F. Lin, P. Lyu, X. Peng, W. Yu, X. Hai, C. Chen,

H. Yang, J. Martin, J. Lu, X. Luo, A. H. Castro Neto, S. J. Pennycook, J. Ding, Y. Feng and J. Lu, Adv. Mater., 2020, 32, 1907645.

30 I. A. Verzhbitskiy, D. Voiry, M. Chhowalla and G. Eda, 2D Mater., 2020, 7, 035013.

31 C. Wang, Q. He, U. Halim, Y. Liu, E. Zhu, Z. Lin, H. Xiao, X. Duan, Z. Feng, R. Cheng, N. O. Weiss, G. Ye, Y.-C. Huang, H. Wu, H.-C. Cheng, I. Shakir, L. Liao, X. Chen, W. A. Goddard Iii, Y. Huang and X. Duan, Nature, 2018, 555, 231-236.

32 Q. He, Z. Lin, M. Ding, A. Yin, U. Halim, C. Wang, Y. Liu, H.-C. Cheng, Y. Huang and X. Duan, Nano Letters, 2019, 19, 6819-6826.

33 N. Wang, H. Tang, M. Shi, H. Zhang, W. Zhuo, D. Liu, F. Meng, L. Ma, J. Ying, L. Zou, Z. Sun and X. Chen, Journal of the American Chemical Society, 2019, 141, 17166-17173.

34 A. R. Wildes, V. Simonet, E. Ressouche, G. J. McIntyre, M. Avdeev, E. Suard, S. A. J. Kimber, D. Lançon, G. Pepe, B. Moubaraki and T. J. Hicks, Phys. Rev. B, 2015, 92, 224408.

35 P. A. Joy and S. Vasudevan, Phys. Rev. B, 1992, 46, 5425-5433.

36 K. Kim, S. Y. Lim, J.-U. Lee, S. Lee, T. Y. Kim, K. Park, G. S. Jeon, C.-H. Park, J.-G. Park and H.

Cheong, Nature Communications, 2019, 10, 345.

37 C. Berthier, Y. Chabre and M. Minier, Solid State Communications, 1978, 28, 327-332. 
38 G. Giunta, V. Grasso, F. Neri and L. Silipigni, Phys. Rev. B, 1994, 50, 8189-8194.

39 E. Manova, A. Leaustic, I. Mitov, D. Gonbeau and R. Clement, Molecular Crystals and Liquid

Crystals Science and Technology. Section A. Molecular Crystals and Liquid Crystals, 1998, 311, 155160.

40 X. Ma, L. Zhang, C. Xu, Q. Dong, R. I. Walton, Z. Li, H. Shi, G. Chen, J. Hu, J. Li and H. Yang, Chem. Commun., 2020, 56, 4603-4606.

41 R. Brec, D. M. Schleich, G. Ouvrard, A. Louisy and J. Rouxel, Inorg. Chem., 1979, 18, 1814-1818.

42 M. Rajapakse, B. Karki, U. O. Abu, S. Pishgar, M. R. K. Musa, S. M. S. Riyadh, M. Yu, G.

Sumanasekera and J. B. Jasinski, npj 2D Mater Appl, 2021, 5, 30.

43 R. Brec, Solid State Ionics, 1986, 22, 3-30.

44 I. Saikh, C. Julien and M. Balkanski, in Solid State Microbatteries, eds. J. R. Akridge and M.

Balkanski, Springer US, Boston, MA, 1990, pp. 297-300.

45 X. Li, Y. Fang, J. Wang, B. Wei, K. Qi, H. Y. Hoh, Q. Hao, T. Sun, Z. Wang, Z. Yin, Y. Zhang, J. Lu, Q. Bao and C. Su, Small, 2019, 15, 1902427.

46 J. Luxa, Š. Cintl, L. Spejchalová, J.-Y. Lin and Z. Sofer, ACS Appl. Energy Mater., 2020, 3, 11992 11999.

47 D. C. Luehrs, R. T. Iwamoto and J. Kleinberg, Inorg. Chem., 1966, 5, 201-204.

48 B. E. Taylor, J. Steger and A. Wold, Journal of Solid State Chemistry, 1973, 7, 461-467.

49 M. Z. Shi, N. Z. Wang, B. Lei, J. J. Ying, C. S. Zhu, Z. L. Sun, J. H. Cui, F. B. Meng, C. Shang, L. K. Ma and X. H. Chen, New J. Phys., 2018, 20, 123007.

50 C. Wan, X. Gu, F. Dang, T. Itoh, Y. Wang, H. Sasaki, M. Kondo, K. Koga, K. Yabuki, G. J. Snyder,

R. Yang and K. Koumoto, Nature Mater, 2015, 14, 622-627.

51 C. Wan, Y. Kodama, M. Kondo, R. Sasai, X. Qian, X. Gu, K. Koga, K. Yabuki, R. Yang and K. Koumoto, Nano Lett., 2015, 15, 6302-6308.

52 D. O'hare and J. S. O. Evans, Comments on Inorganic Chemistry, 1993, 14, 155-206.

53 K. Kim and D. A. Cleary, J. Phys. Chem., 1990, 94, 3816-3819.

54 M. Ormaza, P. Abufager, N. Bachellier, R. Robles, M. Verot, T. Le Bahers, M.-L. Bocquet, N. Lorente and L. Limot, J. Phys. Chem. Lett., 2015, 6, 395-400.

55 C.-T. Kuo, M. Neumann, K. Balamurugan, H. J. Park, S. Kang, H. W. Shiu, J. H. Kang, B. H. Hong, M. Han, T. W. Noh and J.-G. Park, Sci Rep, 2016, 6, 20904.

56 I. Kerrache, C. Julien and C. Sourisseau, Solid State Ionics, 1996, 92, 37-43.

57 G. Ouvrard, E. Prouzet, R. Brec, S. Benazeth and H. Dexpert, Journal of Solid State Chemistry, 1990, 86, 238-248.

58 D. Hartley and M. J. Ware, J. Chem. Soc. A, 1969, 0, 138-142.

59 R. Aroca, M. Nazri, G. A. Nazri, A. J. Camargo and M. Trsic, Journal of Solution Chemistry, 2000, 29, 1047-1060.

60 C. Neumann, S. Reichardt, P. Venezuela, M. Drögeler, L. Banszerus, M. Schmitz, K. Watanabe, T. Taniguchi, F. Mauri, B. Beschoten, S. V. Rotkin and C. Stampfer, Nat Commun, 2015, 6, 8429.

61 B. J. Robinson, C. E. Giusca, Y. T. Gonzalez, N. D. Kay, O. Kazakova and O. V. Kolosov, 2D Mater., 2015, 2, 015005. 
a)

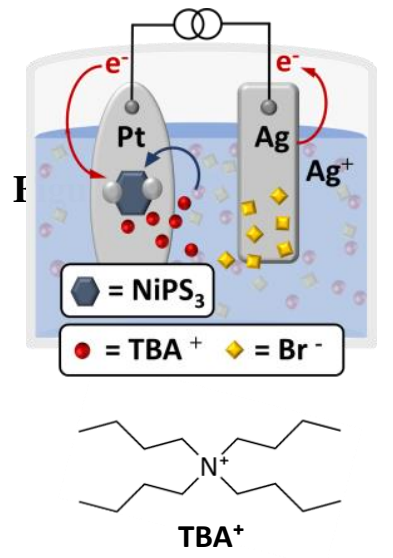

b)

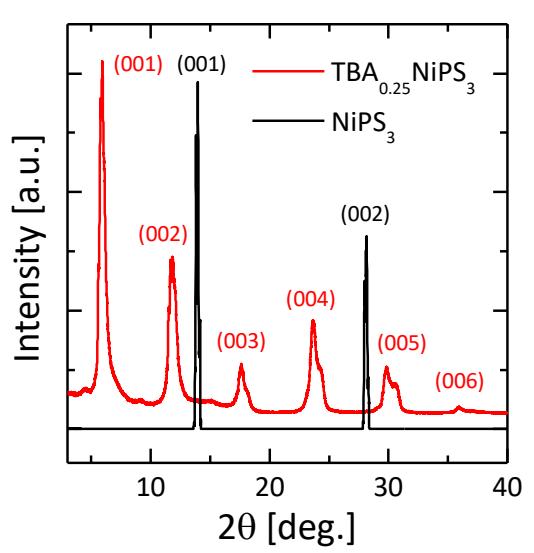

c)

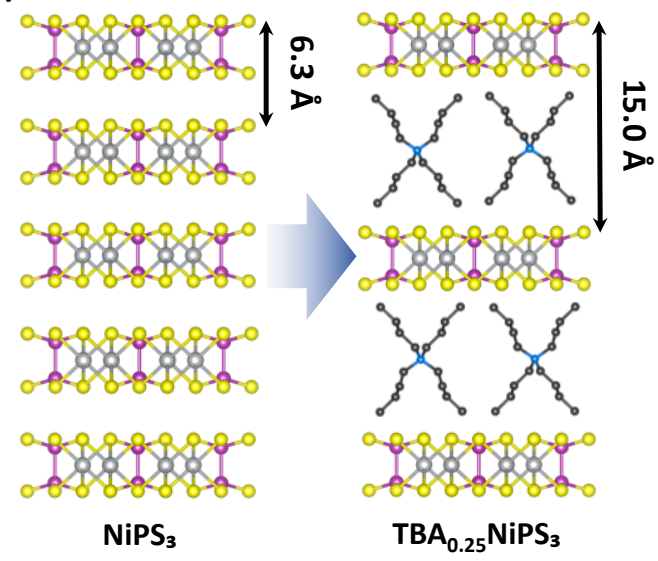

Figure 1: a) Electrochemical setup used for the intercalation of $\mathrm{TBA}^{+}$cations into $\mathrm{NiPS}_{3}$ bulk crystal. The chemical structure of tetrabutylammonium (TBA) cation is also shown. b) X-ray diffraction patterns of pristine $\mathrm{NiPS}_{3}$ (black line) and $\mathrm{TBA}_{0.25} \mathrm{NiPS}_{3}$ (red line). c) Scheme of the intercalation of $\mathrm{TBA}^{+}$cations. During the process, the interlayer distance increases from $6.3 \AA$ to $15.0 \AA$ due to the presence of $\mathrm{TBA}^{+}$.

a)

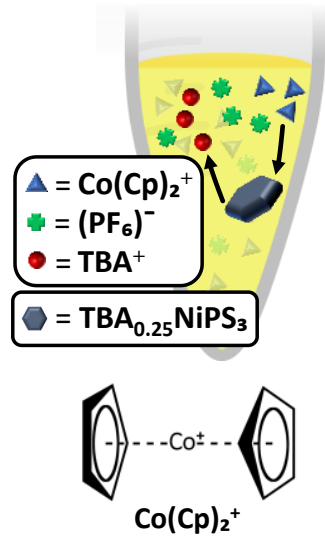

b)

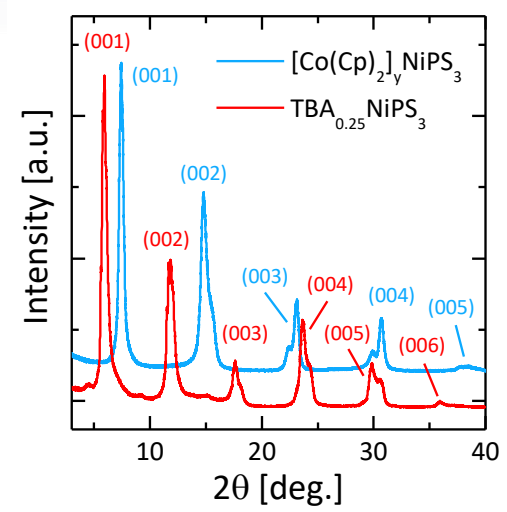

c)

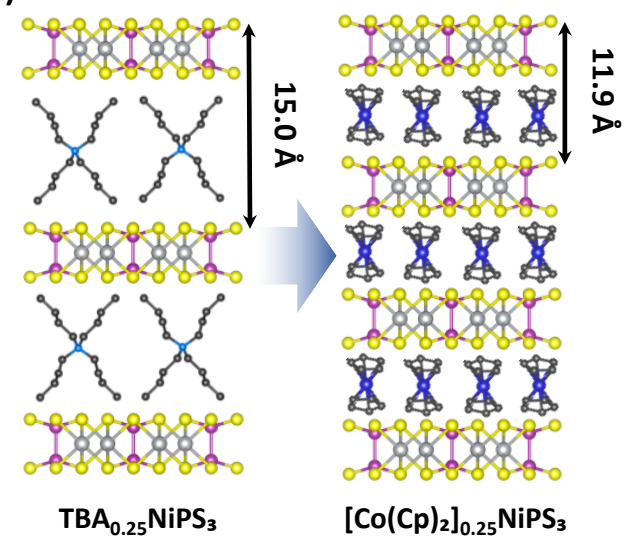

Figure 2: a) Setup for the cation exchange process. A $\mathrm{TBA}_{0.25} \mathrm{NiPS}_{3}$ bulk crystal is immersed in a closed vial containing an acetonitrile solution of $\mathrm{Co}(\mathrm{Cp})_{2} \mathrm{PF}_{6}$. The Chemical structure of Bis-(cyclopentadienyl)cobalt(III), Cobaltocenium $\left(\mathrm{Co}(\mathrm{Cp})_{2}{ }^{+}\right)$is also shown. b) X-ray diffraction patterns of bulk $\mathrm{TBA}_{0.25} \mathrm{NiPS}_{3}$ before and after cation exchange (red and blue lines, respectively). c) Scheme of the cation exchange. During the process, the interlayer distance decreases from $15 \AA$ to $11.9 \AA$ due to the smaller size of $\mathrm{Co}(\mathrm{Cp})_{2}{ }^{+}$in comparison to $\mathrm{TBA}^{+}$. 
a)

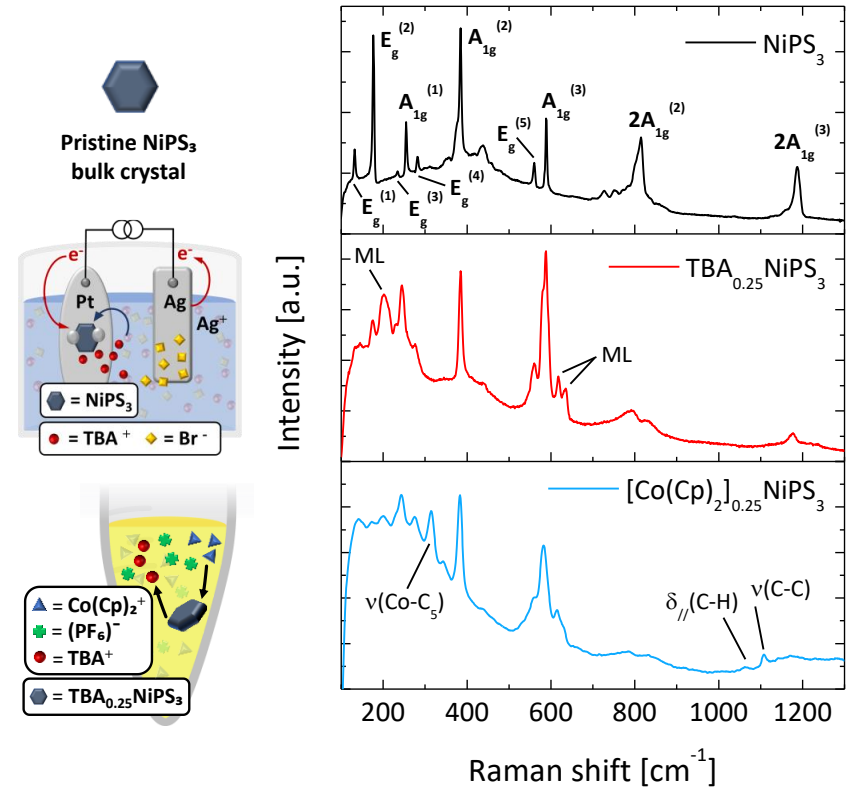

b)

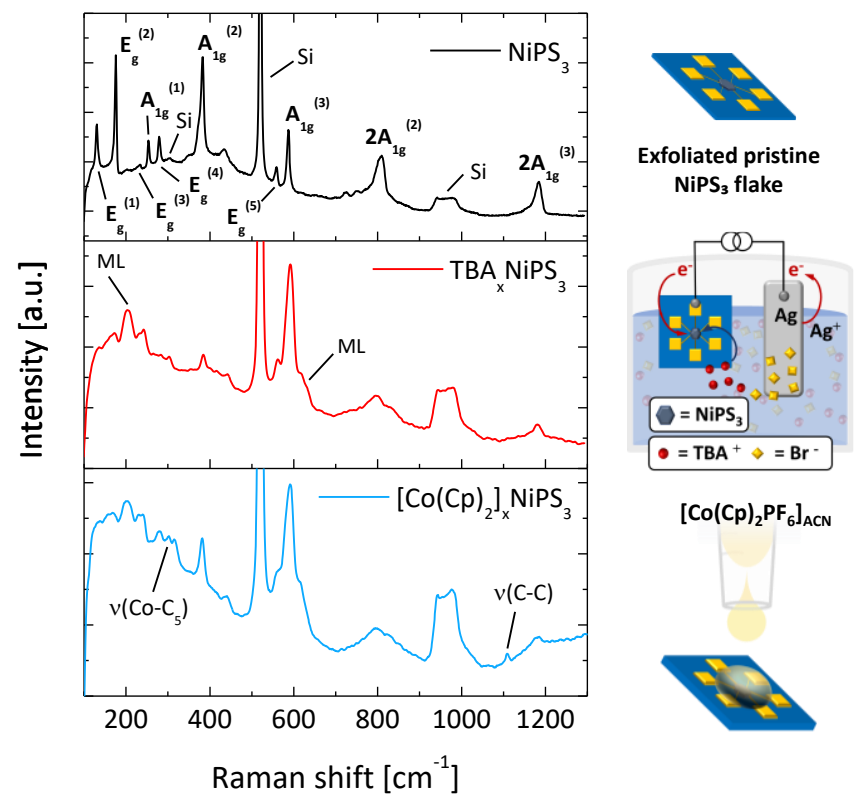

Figure 3: a) Raman spectra of pristine $\mathrm{NiPS}_{3}, \mathrm{TBA}_{0.25} \mathrm{NiPS}_{3}$ and $\left[\mathrm{Co}(\mathrm{Cp})_{2}\right]_{0.25} \mathrm{NiPS}_{3}$ bulk crystals. b) Raman spectra of pristine $\mathrm{NiPS}_{3}, \mathrm{TBA}_{x} \mathrm{NiPS}_{3}$ and $\left[\mathrm{Co}(\mathrm{Cp})_{2}\right]_{0.25} \mathrm{NiPS}_{3}$ flakes. On the left and right side, we display the schematics corresponding to each intercalation step.

a)

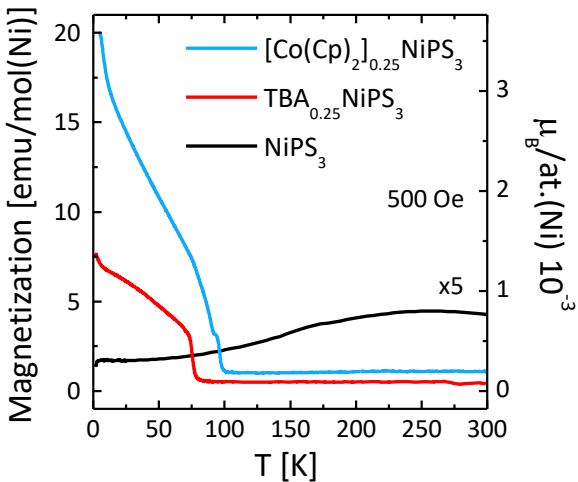

c) Pristine $\mathrm{NiPS}_{3}$

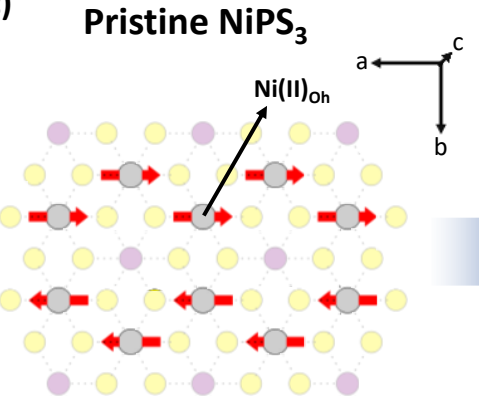

b)

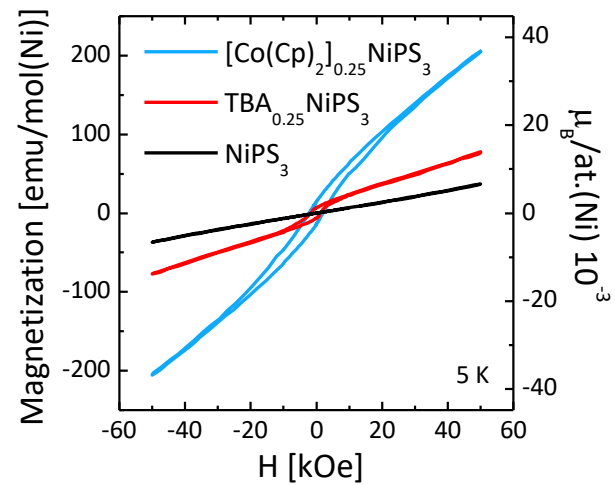

Intercalated $\mathrm{NiPS}_{3}$

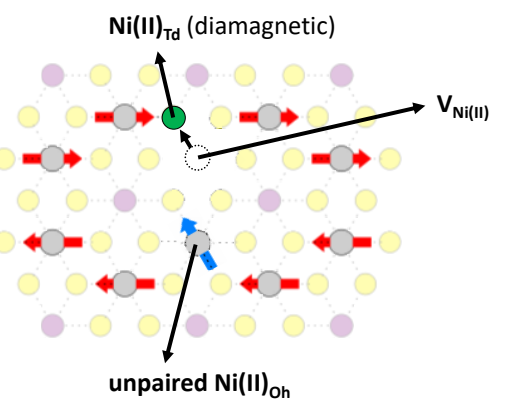

Figure 4: a) Field-cooled molar magnetization vs. temperature for bulk $\mathrm{NiPS}_{3}, \mathrm{TBA}_{0.25} \mathrm{NiPS}_{3}$ and $\left[\mathrm{Co}(\mathrm{Cp})_{2}\right]_{0.25} \mathrm{NiPS}_{3}$ crystals. The applied filed $(500 \mathrm{Oe})$ is oriented parallel to the ab plane of the crystal. b) Hysteresis loops at $5 \mathrm{~K}$ of bulk pristine $\mathrm{NiPS}_{3}, \mathrm{TBA}_{0.25} \mathrm{NiPS}_{3}$ and $\left[\mathrm{Co}(\mathrm{Cp})_{2}\right]_{0.25} \mathrm{NiPS}_{3}$ crystals. c) Scheme of the structural change in the $\mathrm{NiPS}_{3}$ layer accompanying the reduction of the $\mathrm{Ni}$ atoms. 